\title{
Progress: Targeted Therapy, Immunotherapy, and New Chemotherapy Strategies in Advanced Triple-Negative Breast Cancer
}

This article was published in the following Dove Press journal: Cancer Management and Research

\section{Jinhong Shi (D) \\ Feiqi Liu \\ Yanqiu Song (DD}

Cancer Center, The First Hospital of Jilin University, Changchun, Jilin, People's Republic of China
Correspondence: Yanqiu Song

The First Hospital of Jilin University, No.

I, Xinmin Street, Changchun, Jilin I3002 I,

People's Republic of China

Tel +86 18934749569

Email song_yq@jlu.edu.cn

\begin{abstract}
Triple-negative breast cancer (TNBC) is one of the most aggressive subtypes of breast cancer, accounting for approximately $15 \%$ of cases, and is defined by the lack of expression of hormone receptors (estrogen and progesterone receptors) and lack of amplification or overexpression of human epidermal growth receptor 2 (HER2). Due to the lack of targets of hormone receptors and HER2, treatment of TNBC or advanced TNBC relies on conventional chemotherapeutic agents, but their efficacy and prognosis are poor. In patients with advanced TNBC, poorer outcomes are observed. Recently, with the launch of clinical trials and advancements in molecular studies, targeted therapy for signaling transduction pathways, immunotherapy for immune checkpoints, and new chemotherapy strategies have provided feasible or potential therapeutic options for advanced TNBC. This review aimed to summarize recent progress in targeted therapy, immunotherapy, and chemotherapy for advanced TNBC.
\end{abstract}

Keywords: advanced TNBC, targets, immune checkpoint inhibitor, refractory

\section{Introduction}

According to the American Cancer Society statistics from 1975 to 2016, breast cancer continued to have the highest incidence among tumors in women in the United States. The agency estimated that, in 2020, morbidity and mortality of breast cancer accounted for $30 \%$ and $15 \%$, respectively, of all tumors in women. ${ }^{1}$ Based on immunohistochemistry (IHC) and fluorescence in situ hybridization analysis, triple-negative breast cancer (TNBC) is one of the subtypes of breast cancer defined by the lack of expression of hormone receptors, including estrogen receptor (ER) and progesterone receptor (PR), and lack of amplification or overexpression of human epidermal growth factor receptor 2 (HER2). ${ }^{2}$ TNBC accounts for approximately $15 \%$ of all breast cancers. ${ }^{3}$ TNBC is characterized by younger onset age, high risk of recurrence after surgery, increased risk of visceral metastasis, and poor prognosis, which is a challenging problem in clinical practice. ${ }^{4,5}$ Due to the absence of therapeutic targets of hormone receptors and HER2, the main treatment for TNBC is chemotherapy, but benefits from traditional chemotherapy are poor. In the advanced stage of TNBC (ie, locally advanced or metastatic), the selectable drugs are further reduced. In the past few years, the overall survival (OS) for advanced TNBC had not significantly improved, and the median duration from distant recurrence to eventual death was generally 14-15 months, which was 
significantly shorter than that of other subtypes of breast cancer. ${ }^{6}$ Therefore, it is essential to identify new strategies for advanced TNBC.

TNBC is a highly molecular heterogeneous disease that can be stratification furtherly. Lehmann et al initially classified TNBC into six subtypes - basal-like 1 (BL1), basallike 2 (BL2), immunomodulatory (IM), mesenchymal (M), mesenchymal stem-like (MSL), and luminal androgen receptor (LAR). The main characteristics of the above subtypes are summarized briefly as follows: BL1 is enriched in genes involved in pathways of cell cycle and DNA damage response, such as mutations in the breast cancer susceptibility genes (BRCA); BL2 contains genes involved in growth factor signaling and receptors, such as epidermal growth factor receptors (EGFRs); IM contains genes involved in angiogenesis, such as vascular endothelial growth factor receptor (VEGFR) 2, and immune cell processes; the $\mathrm{M}$ and MSL subtypes contain various unique genes involved in cell motility and differentiation pathways, such as mutation in the phosphatidylinositol4,5-bisphosphate 3-kinase catalytic subunit alpha (PIK3CA), and the phosphoinositide 3-kinase (PI3K)/protein kinase $\mathrm{B}(\mathrm{AKT}) /$ mammalian target of rapamycin (mTOR) pathways. ${ }^{7}$ MSL is unique in that it is enriched with genes involved in angiogenesis, immune signaling, and growth factor pathways; LAR is ER-negative/ARpositive and contains genes enriched in hormonally regulated pathways. Relapse-free survivals (RFSs) were significantly different between the subtypes $(p=0.0083)$, regardless of regimens or duration of treatment. The LAR subtype had a shorter RFS than the BL1, IM, and MSL subtypes $(p<0.05)$. A possible explanation for this may be that the LAR subtype is diagnosed at an older age compared with other subtypes $(\mathrm{p}<0.0001){ }^{2}$

\section{Targeted Therapy Poly ADP-Ribose Polymerase (PARP) Inhibitors}

BRCA are tumor suppressor genes that repair fractured double-stranded DNA by homologous recombination repair. Germline BRCA1/2 mutations cause defects in DNA repair and homologous recombination repair, thus inducing instability in the genome. ${ }^{8}$ This mechanism is the basis of tumorigenesis. Patients with breast cancer carrying BRCA1/2 mutations account for $5-10 \%$ of breast cancer cases. Such mutations are more likely found in patients with a family history of breast or ovarian cancer (particularly with first-degree family history), younger patients (age $<45$ years), and patients with TNBC., ${ }^{9,10}$ Approximately $40 \%$ of patients with TNBC carry BRCA1/2 mutations, and approximately $60 \%$ of the BRCA1-mutated cancers present $\mathrm{TNBC},{ }^{11}$ indicating that TNBC and BRCA1/2 mutations are correlated but not completely coincidental.

The poly ADP-ribose polymerase (PARP) family consist of ribozymes that catalyze the ribosylation of ADP, including approximately 17 proteins. These family members engage in base resection and repair, thus exerting a crucial effect in repairing single-stranded DNA damage. ${ }^{12}$ If we use PARP inhibitors for BRCA1/2-mutated cells, these two repair pathways, single-stranded and doublestranded DNA repair, are blocked. In addition, a study demonstrated that BRCA2-deficient cells are sensitive to PARP1 inhibitors. ${ }^{13}$ These two statements are currently considered the theoretical basis for using PARP inhibitors in the treatment of BRCA1/2-mutated breast cancers. The interaction between BRCA and PARP significantly conforms to the concept of "synthetic lethality," according to which there is no effect when there is a defect in one of the two genes, but a combination of two deficient genes results in cell or organism death. ${ }^{14}$ Synthetic lethality is a defect in tumor suppressor genes in principle and is specifically lethal to tumor cells, but it has no effect on normal cells. ${ }^{15}$ This mechanism is conducive for precisely killing tumor cells.

Olaparib, an oral PARP inhibitor, can suppress PARP1, PARP2, and PARP3 simultaneously. It is effective to treat patients with breast cancer with either BRCA1 or BRCA2 mutations. The OlympiAD trial was designed to compare olaparib with treatment of physician's choice (TPC) in patients with HER2-negative metastatic breast cancer (MBC) and germline BRCA mutation. This trial demonstrated that olaparib significantly prolonged median progression-free survival (PFS) compared with TPC (7.0 months vs 4.2 months; $p<0.001)$. In the olaparib group, most treatment-related adverse events (TRAEs), including nausea, anemia, vomiting, fatigue, and neutropenia, were low grade. There is also a lower rate of grade $\geq 3$ TRAEs in the olaparib group than in the TPC group. ${ }^{16}$ Subsequent OS analysis showed that there was no statistically significant difference between the two groups. However, the subgroup analysis showed that patients who received olaparib as first-line therapy were likely to obtain OS benefit. There was no cumulative effect of long-term toxicity in the olaparib group (Table 1). ${ }^{17}$ 
Table I Main PARP/AKT Inhibitors in MBC/TNBC

\begin{tabular}{|c|c|c|c|c|c|}
\hline Drug & Trial & Phase & Population & Design & PFS \\
\hline \multicolumn{6}{|c|}{ PARP inhibitors } \\
\hline Olaparib & OlympiAD & 3 & gBRCA-muted MBC & Olaparib vs TPC & $\begin{array}{l}7.0 \text { vs } 4.2 \text { months } \\
\text { (HR } 0.58 ; p<0.00 I)\end{array}$ \\
\hline Talazoparib & EMBRACA & 3 & gBRCA-muted MBC & Talazoparib vs TPC & $\begin{array}{l}8.6 \text { vs } 5.6 \text { months } \\
(H R \quad 0.54 ; p<0.00 I)\end{array}$ \\
\hline Veliparib & BROCADE & 2 & gBRCA-muted MBC & $\begin{array}{l}\text { VCP vs PCP } \\
\text { VT vs PCP }\end{array}$ & $\begin{array}{l}\text { I4.I vs } 12.3 \text { months } \\
(p=0.227) ; \\
7.4 \text { vs I } 2.3 \text { months } \\
(\text { HR I.858; } p=0.00 I)\end{array}$ \\
\hline \multicolumn{6}{|c|}{ AKT inhibitors } \\
\hline Ipatasertib & LOTUS & 2 & Advanced TNBC & IPAT + PAC vs PBO + PAC & $\begin{array}{l}\text { ITT: } 6.2 \text { vs. } 4.9 \text { months } \\
\text { (HR } 0.60 ; p=0.037) \\
\text { PTEN-low: } 6.2 \text { vs. } 3.7 \text { months }(p=0 \cdot 18)\end{array}$ \\
\hline Capivasertib & PAKT & 2 & Advanced TNBC & $\mathrm{CAPI}+\mathrm{PAC}$ vs $\mathrm{PBO}+\mathrm{PAC}$ & $\begin{array}{l}5.9 \text { vs. } 4.2 \text { months } \\
(H R \text { 0.74; } p=0.06 *)\end{array}$ \\
\hline
\end{tabular}

Note: *Predefined significance level, one-sided $\mathrm{p}=0.10$.

Abbreviations: gBRCA, germline BRCA; m, months; VCP, ipatasertib + carboplatin + paclitaxel; VT, ipatasertib + temozolomide; PCP, placebo + carboplatin + paclitaxel; PAC, paclitaxel; IPAT, ipatasertib; PAC, paclitaxel; PBO, placebo; ITT, intention-to-treat; CAPI, capivasertib.

Talazoparib is an oral and selective inhibitor of PARP1/2. The EMBRACA trial compared talazoparib with TPC (capecitabine, eribulin, gemcitabine, or vinorelbine) in patients with MBC and germline BRCA mutations. The study demonstrated that the talazoparib arm significantly prolonged median PFS compared to the control arm (8.6 months vs 5.6 months; $\mathrm{p}<0.001)$. The objective response rate (ORR) was higher in the talazoparib group than in the TPC group $(62.6 \%$ vs $27.2 \%$; $\mathrm{p}<0.001)$. The most common TRAEs in the talazoparib group were hematologic toxicities (primarily anemia). The incidence of grade 3 or 4 hematologic TRAEs was higher in the talazoparib group than in the TPC group $(55 \%$ and $38 \%$, respectively). ${ }^{18}$ Using the European Organization for Research and Treatment of Cancer Quality of Life Questionnaire Core 30 and its breast cancer module, QLQ-BR23, researchers demonstrated that patients who received talazoparib had an overall improvement in quality of life and significant delay in the time to definitive clinically meaningful deterioration. ${ }^{19}$ In addition, patients with germline BRCA mutation who received and those who did not receive platinum therapy both benefited from talazoparib treatment (Table 1). ${ }^{20}$

Veliparib is an oral PARP1/2 inhibitor. Presently, recommended dosing schedules of veliparib in various combination regimens are different. A Phase 1 trial evaluated the addition of veliparib to cisplatin and vinorelbine in advanced TNBC and/or BRCA mutation-associated breast cancer. This trial found that patients with germline BRCA mutation were most likely to benefit from study therapy, regardless of the veliparib dose, and veliparib 300 mg twice daily (BID) schedule was well tolerated. ${ }^{21}$ The BROCADE Phase 2 trial evaluated the addition of veliparib to carboplatin/paclitaxel (VCP) or temozolomide (VT) in patients with BRCA-mutated MBC. Eligible patients were randomized 1:1:1 to VCP (veliparib at 120 mg BID, days 1-7, 3-week cycle), VT, or placebo plus carboplatin/paclitaxel (PCP). Compared to PCP, VCP significantly improved ORR $(77.8 \%$ vs $61.3 \% ; p=0.027)$ but did not significantly improve PFS and OS. Compared to PCP, VT did not significantly improve ORR, PFS, and OS. ${ }^{22}$ The corresponding Phase 3 trial (BROCADE3, NCT02163694) has completed recruitment and evaluates VCP versus PCP in HER2-negative BRCA-associated MBC. The results have not been reported thus far. An exposure-response analysis supported that veliparib dosing of the abovementioned BROCADE trial exhibited additional benefit without significantly affecting safety when combined with carboplatin and paclitaxel. A higher dose of veliparib was not observed to have a greater 
benefit in this combination in the abovementioned patients. ${ }^{23}$ A phase 1 trial evaluated the combination of carboplatin and veliparib at intermittent or continuous schedules in MBC. The combination veliparib continuous schedule (250 mg twice daily, d1-d21) and carboplatin (area under the curve 5, 3-week cycle) demonstrated antitumor activity and good tolerance (Table 1). ${ }^{24}$

Currently, there are four Food and Drug Administration (FDA)-approved PARP inhibitors-olaparib, talazoparib, rucaparib, and niraparib. Only olaparib and talazoparib have been approved for patients with the germline BRCA mutation and HER2-negative MBC. The remaining two PARP inhibitors are indicated for advanced ovarian cancer.

\section{AKT Inhibitors}

AKT is an important member of the PI3K/AKT/mTOR signaling pathway. ${ }^{25}$ AKT is a key node of various downstream signaling pathways and promotes cell survival, growth, metabolism, invasion, and migration by acting on downstream signaling molecules. ${ }^{26,27}$ PIK3CA or AKT1 mutation, and phosphatase and tensin homolog (PTEN) inactivating or loss can activate PI3K/AKT/ mTOR pathway, which is common in TNBC. ${ }^{28,29}$ The structure and activating mechanism of AKT have been found for nearly 30 years, but many studies on AKT inhibitors had come up short until the completion of the LOTUS trial and PAKT trial.

The LOTUS trial evaluated the safety and efficacy of adding ipatasertib (an oral AKT inhibitor) to paclitaxel as a first-line therapy for advanced TNBC. Patients received ipatasertib plus paclitaxel or placebo plus paclitaxel. The median PFS was 6.2 months in the ipatasertib group versus 4.9 months in the placebo group (hazard ratio [HR], 0.60; $95 \%$ confidence interval [CI], 0.37-0.98; $\mathrm{p}=0.037$ ). Within the PIK3CA/AKT1/PTEN-altered subgroup, the difference in the median PFSs was more significant (9.0 months and 4.9 months, respectively; $p=0 \cdot 041$ ). Within the PIK3CA/ AKT1/PTEN-non-altered subgroup, there was no statistically significant difference between the two groups $(\mathrm{p}=0 \cdot 36)$. The most common TRAEs in the ipatasertib group were gastrointestinal toxicities (diarrhea, nausea, and vomiting), alopecia, neuropathy, fatigue, and rash with low grade. The rate of grade $\geq 3$ TRAEs in the ipatasertib group was $54 \%$, consisting mainly of diarrhea $(23 \%) .{ }^{30}$ The updated OS analysis of the LOTUS trial showed that an improvement trend was observed in the ipatasertib group. The 1-year OS rate in the ipatasertib group was $83 \%$, while that in the placebo group was $70 \%$ (Table 1$){ }^{31}$

Similarly, the PAKT trial on paclitaxel plus AKT inhibitor capivasertib versus paclitaxel plus placebo as firstline therapy in advanced TNBC also yielded a positive result. The capivasertib group had significantly prolonged median PFS and OS compared to the placebo group. The median PFSs in the two groups were 5.9 months and 4.2 months, respectively (one-sided $\mathrm{p}=0.06$ ). The median OSs in the two groups were 19.1 months and 12.6 months, respectively ( $\mathrm{HR}, 0.61 ; 95 \% \mathrm{CI}, 0.37-0.99 ; \mathrm{p}=0.04)$. In the PIK3CA/AKT1/PTEN-altered subgroup, the difference in the median PFSs was more significant (9.3 months vs 3.7 months, respectively; $\mathrm{p}=0.01$ ). The most common grade $\geq 3$ TRAE in the capivasertib group was diarrhea $(13 \%)$ (Table 1). ${ }^{32}$

\section{AR Inhibitors}

AR, a member of the nuclear steroid hormone receptor family, was found in approximately $60-70 \%$ of all breast cancers and $20-40 \%$ of TNBCs. ${ }^{33,34}$ Lehmann et al noted that AR mRNA was highly expressed in the LAR subtype, on an average, at nine-fold higher than in the other subtypes. Growth of the LAR subtype is deemed to be driven by the AR signaling pathway. ${ }^{2}$ Targeting AR therapy is a new strategy for TNBC. In addition, LAR subtype is rich in PIK3CA mutations and thus sensitive to PI3K/mTOR inhibitors. ${ }^{2,35}$ Patients with AR-negative breast cancer are observed to have generally younger diagnostic age compared to those with AR-positive breast cancer. ${ }^{36}$ In early breast cancer, AR expression is considered to be associated with better prognosis than those without AR expression. ${ }^{37}$ Therefore, some scholars have proposed the concept of "Quadruple-negative breast cancer," which is defined as AR/ER/PR/HER2-negative breast cancer. ${ }^{38,39}$ However, the prognostic significance of AR in TNBC is still controversial. ${ }^{40,41}$

Bicalutamide is an oral, nonsteroidal AR inhibitor that is initially combined with luteinizing hormone-releasing hormone analogues for the treatment of metastatic prostate cancer. Recently, bicalutamide showed antitumor activity and ER/PR-negative, AR-positive ( $>10 \%$ IHC staining) MBC with a $19 \%$ clinical benefit rate (CBR) and 3month median PFS. No patients who received bicalutamide had grade 4/5 TRAEs. ${ }^{42}$ Abiraterone acetate (AA) inhibits 17-[ $\alpha$ ]-hydroxylase/17,20-lyase (CYP17), leading to reduced serum androgen levels. ${ }^{43} \mathrm{AA}$ is often used in castration-resistant prostate cancer. ${ }^{44}$ Bonnefoi et al 
reported that AA plus prednisone exhibited 20\% CBR and 2.8-month median PFS in AR-positive TNBC. The most common TRAEs in this trial were fatigue, hypertension, hypokalaemia, and nausea with low grade. ${ }^{45}$ Enzalutamide is an oral, more powerful AR inhibitor that acts on multiple steps in the AR signaling pathway. Enzalutamide demonstrated antitumor activity and good tolerance among patients with metastatic AR-positive tumors. Traina et al reported that enzalutamide $160 \mathrm{mg}$ daily exhibited 33\% CBR at 16 weeks and 28\% CBR at 24 weeks in patients with advanced AR-positive TNBC. The median PFS and OS were 3.3 months and 17.6 months, respectively, in the evaluable subgroup. Moreover, 3\% patients who received enzalutamide had grade $\geq 3$ TRAEs, and they experienced only fatigue (Table 2). ${ }^{46}$

\section{Angiogenesis Inhibitors}

Most studies have focused on angiogenesis inhibitors including vascular endothelial growth factor (VEGF) and VEGFR targets in the treatment of TNBC. The efficacy of anti-angiogenesis monotherapy in advanced TNBC is limited. ${ }^{47}$ However, addition of angiogenesis inhibitors to conventional chemotherapy has shown a feasible antitumor effect. The ECOG 2100 trial demonstrated that paclitaxel plus bevacizumab (an anti-VEGF monoclonal antibody) compared with paclitaxel alone as first-line treatment significantly improved median PFS in patients with metastatic HER2-negative breast cancer (11.8 months vs 5.9 months, $\mathrm{p}<0.001){ }^{48}$ Based on this trial, bevacizumab was approved by the FDA in 2008. A meta-analysis of the E2100, AVADO, and RIBBON trials showed that compared with chemotherapy alone, the combination of bevacizumab and taxane-based chemotherapy for advanced TNBC patients improved the median PFS by 2.7 months $(\mathrm{p}<0.0001)$. However, the combination group did not have any OS benefit. The most common grade $\geq 3$ TRAEs with bevacizumab-containing regimens were neutropenia, sensory neuropathy, and hypertension. ${ }^{49}$

In recent years, some optimized combination regimens have also shown satisfactory outcomes in advanced TNBC -weekly paclitaxel and carboplatin plus bevacizumab: 10.3-month median PFS and 25.7-month median $\mathrm{OS}^{50}$ and nab-paclitaxel plus bevacizumab followed by bevacizumab and erlotinib (anti-EGFR) maintenance therapy: 9.1-month median PFS, 18.1-month median OS, and $74 \%$ partial response rate. $^{51}$ In addition, long ncRNA encoded 60-amino acid polypeptide (ASRPS), centromere protein $\mathrm{U}$, endogenous hydrogen sulfide, and exosomal- annexin A2 have been found to be associated with angiogenesis and suggested to be potential targets. ${ }^{52-55}$

\section{Potential Therapeutic Targets and Strategies}

Palbociclib is an oral and highly selective cyclin-dependent kinase 4/6 (CDK4/6) inhibitor that has been approved by the FDA for the treatment of patients with hormone receptor-positive and HER2-negative advanced breast cancer. Retinoblastoma protein $(\mathrm{pRb})$ is a major phosphorylated substrate of $\mathrm{CDK} 4 / 6$ and a key response to CDK4/6 inhibitors. ${ }^{56} \mathrm{~A}$ preclinical study revealed that palbociclib and the dual mTOR inhibitor MLN0128 had synergistic anticancer activity in both pRb-expressing ER-negative breast cancer and TNBC patient-derived xenograft model. ${ }^{57}$ This study found a new target of $\mathrm{TNBC}-\mathrm{pRb}$ and implied the use of palbociclib in breast cancer (Table 3 ). However, in patients receiving palbociclib treatment, resistance is common. ${ }^{58}$ Researchers have demonstrated that enzalutamide could reverse the resistance of palbociclib both in vitro and vivo. ${ }^{59}$ An ongoing trial (NCT02605486) evaluated the combination of enzalutamide and palbociclib for the treatment of AR-positive advanced TNBC (Table 2). ${ }^{60}$ We expect a new strategy, dual-targeting AR and CDK4/6 in TNBC.

Johnstone et al established four metastatic capacity models of TNBC, within the MDA-MB-231_HM model, which was characterized by high metastasis to the lung, liver, spleen, and spine. Fibroblast growth factor 13 (FGF13) is highly expressed in the HM model and correlated with metastasis of TNBC. ${ }^{61}$ FGF13 is a potential target for blockade of TNBC metastasis. Fibroblast growth factor receptors (FGFRs) are transmembrane receptor families of FGFs. FGFR3 was highly expressed in TNBC cell lines prepared by researchers and carried FGFR3TACC3 gene fusion. Public dataset analysis showed that FGFR3 overexpression was associated with poor OS in breast cancer. However, the frequency was low and only FGFR3-TACC3 gene fusion activated the oncogenic driver function of FGFR3 (Table 3). ${ }^{62}$

Moesin (MSN), a member of the ezrin-radixin-moesin family, plays a role in the regulation of cell growth, proliferation, invasion, and metastasis. ${ }^{63} \mathrm{MSN}$ was highly expressed in TNBC compared with other subtypes of breast cancer and correlated with poor OS. MSN enters the nucleus with the help of nucleoprotein NONO and activates downstream cyclic adenosine 3.5-monophosphate (cAMP) 
Table 2 Clinical Trials: Targeting AR Therapy in TNBC

\begin{tabular}{|l|l|l|l|l|l|}
\hline Trial & Phase & Pts & Regimen & CBR (\%) & PFS (Months) \\
\hline NCT004687I5 & 2 & 424 & Bicalutamide 150 mg daily & 19 & 3.0 \\
NCT0184232I & 2 & 30 & AA 1000 mg daily + prednisone 5 mg twice daily & 20 & 2.8 \\
NCT01889238 & 2 & 78 & Enzalutamide 160 mg daily & 28 & 3.3 \\
NCT02605486 & $1 / 2$ & $5 \mathrm{I}$ & Palbociclib 100 mg daily + bicalutamide I00 mg daily, 3 weeks on I week off & NR & NR \\
\hline
\end{tabular}

Abbreviations: Pts, patients; NR, not reported.

Table 3 Potential Targets in TNBC

\begin{tabular}{|l|l|l|}
\hline Targets & Characteristic & Regimen \\
\hline PRb & Substrate of CDK4/6 & Palbociclib + MLN0I28 \\
FGFI3 & Overexpresses in the HM model of TNBC & Unclear FGFI3 inhibitor \\
FGFR3 & Correlates with poor OS & Unclear \\
FGFR3-TACC3 fusion gene & Activates oncogenic driver function of FGFR3 & Unclear \\
MSN, NONO, CREB & MSN correlates with poor OS & MSN/NONO inhibitor, CREB inhibitor + docetaxel \\
WEEI, ATR & Synergistic effect in TNBC & WEEI inhibitor AZDI775 + ATR inhibitor AZD6738 \\
Src tyrosine kinase & Regulating osteoclastic bone & Dasatinib \\
\hline
\end{tabular}

Abbreviations: pRb, retinoblastoma protein; CDK4/6, cyclin-dependent kinase 4/6; FGFI3, fibroblast growth factor I3; FGFR3, fibroblast growth factor receptor 3; MSN, moesin; CREB, element-binding protein; ATR, rad3-related protein.

response element-binding protein (CREB). Targeting MSN, NONO or CREB suppressed the growth of breast tumor cells in vivo. Additionally, the combination of the CREB inhibitor 666-15 and docetaxel exhibited a better suppressing effect (Table 3). ${ }^{64}$

Most TNBCs harbor p53 deficiency, and thus, they are highly dependent on the cell cycle $\mathrm{G} 2 / \mathrm{M}$ checkpoint to respond to DNA damage. ${ }^{65}$ Tyrosine kinase WEE1 engages in the $\mathrm{G} 2 / \mathrm{M}$ checkpoint and regulates DNA synthesis. ${ }^{66}$ In ataxia telangiectasia, the rad3-related protein (ATR), a member of the phosphatidylinositol 3kinase-related kinase family, plays a key role in DNA damage response. ${ }^{67}$ Researchers have found that a combination treatment of WEE1 inhibitor AZD1775 and ATR inhibitor AZD6738 has synergistic effect in TNBC cell lines. In other words, AZD6738 can enhance AZD1775induced growth inhibition in TNBC by repressing DNA damage repair and excessive replication stress. ${ }^{68}$ These findings inspire further examination of a potential target strategy in TNBC (Table 3).

Src tyrosine kinase plays key role in the regulation of osteoclastic bone resorption. ${ }^{69}$ Lehmann et al found that the mesenchymal-like TNBC subtypes ( $\mathrm{M}$ and MSL), which contain the MDA-MB-231 cell model, were sensitive to the Src inhibitor dasatinib. ${ }^{2}$ Heilmann et al designed an osteotropic clone of MDA-MB-231 cells that simulated a model for bone metastasis in TNBC. Based on the above research findings, dasatinib can inhibit bone metastasis of the cells. ${ }^{70}$ Therefore, it is feasible to use dasatinib to treat bone metastasis of the mesenchymal-like subtypes (Table 3). Other preclinical research studies have shown synergistic interactions in TNBC therapy between dasatinib and afatinib (an EGFR inhibitor), olaparib, and salinomycin (a polyether antibiotic). These interactions should be further verified. ${ }^{71-73}$

\section{Immunotherapy}

Immune checkpoints are protective molecules in the immune system that prevent normal tissue damage caused by over-activation of $T$ cells. In the treatment of breast cancer, the most widely studied immune checkpoint receptors include programmed death 1 (PD-1), and its ligand PD-L1. PD-1 is expressed on activated T cells, B lymphocytes, and natural killer cells and associated with tumor immune resistance. The combination of PD-1 with PD-L1 blocks $\mathrm{T}$ cell proliferation and response. ${ }^{74}$ Breast cancer was previously thought to be a weakened immunogenic tumor, but researchers found that the IM subtype, a type of $\mathrm{TNBC}$, is rich in genes involved in immune cell processes, including immune cell signaling, cytokine signaling, antigen processing and presentation, and signaling through core immune signal transduction pathways. ${ }^{2}$ Second, high average mutation load and tumor-infiltrating lymphocyte (TIL) levels are observed in TNBC, implying high 
immunogenicity in TNBC. ${ }^{75}$ In addition, a study demonstrated that PD-L1 was expressed in both tumor cells and tumor-associated inflammatory cells (eg, immune cells, stromal TILs, and CD8+ T cells) of breast cancer. PD-L1 expression is prevalent among high-grade, hormone receptor-negative breast cancers. Particularly, TNBC has a higher rate of PD-L1 expression than other breast cancer types. $^{76-78}$ These characteristics suggest immune checkpoint inhibitor (ICI) as a feasible therapeutic agent for TNBC.

\section{Monotherapy}

ICI monotherapy demonstrated antitumor activity in the treatment of advanced $\mathrm{TNBC}$, but their response rates widely varied. In terms of ICI first-line therapy in advanced TNBC, pembrolizumab (anti-PD-1 antibody) showed an ORR of $18.5 \%$ or $21.4 \%$ in PD-L1+ subgroup depending on the study. ${ }^{79,80}$ Atezolizumab (anti-PD-L1 antibody) showed $24 \%$ ORR. ${ }^{81}$ However, ICI as second or higher line therapy in advanced TNBC received low ORRs, just $6 \%$ or $5.3 \% .{ }^{81,82}$ A trend toward a higher ORR was observed in the first-line therapy of PD-1/PD-L1 inhibitors.

The PFS of ICI monotherapy in advanced TNBC is generally $1.4-2.1$ months. The OS is generally 9-18 months. The incidence of TRAEs is approximately $60 \%$. The incidence of grade $\geq 3$ TRAEs is generally $10-16 \%$. The most common TRAEs are fatigue, nausea, and diarrhea. $^{79-85}$ Generally, ICI monotherapy was effective but not significant for advanced TNBC. Therefore, most studies currently focus on combination therapy (Table 4).

\section{Combination Therapy}

In the IMpassion 130 trial, atezolizumab plus nab-paclitaxel significantly prolonged median PFS than placebo plus nab-paclitaxel in advanced TNBC (7.2 months and 5.5 months, respectively; $\mathrm{p}=0.002$ ). In the $\mathrm{PD}-\mathrm{L} 1+$ immune cells (ICs) (>1\%) subgroup, the difference in PFSs between the atezolizumab and control groups was more significant (7.5 months and 5.0 months, respectively; $\mathrm{p}<0.001)$. However, there was no significant difference in the OSs between the two groups in the first or second interim analyses. However, in the PD-L1+ subgroup, the first interim analysis showed that the median OS was improved from 15.5 months in the control group to 25.0 months in the atezolizumab group. The second interim analysis showed that the median OS improved from 18.0 months in the control group to 25.0 months in the atezolizumab group. ${ }^{86,87}$ Based on the IMpassion 130 trial, the FDA approved combining atezolizumab with nab-paclitaxel in patients with advanced TNBC with PDL1 ICs $>1 \%$. In a phase $1 \mathrm{~b}$ trial, patients with advanced TNBC received atezolizumab plus nab-paclitaxel. The ORR was $39.4 \%$. The median duration of response was 9.1 months. The median PFS and OS were 5.5 months and 14.7 months, respectively. However, the TRAEs of this regime were obvious. Moreover, $73 \%$ of patients had grade $\geq 3$ TRAEs, neutropenia, and decreased neutrophil level (Table 4). ${ }^{88}$ In contrast to the IMpassion 130 trial, the IMpassion 131 trial (NCT03125902) evaluates atezolizumab plus solvent-based paclitaxel as first-line therapy for advanced TNBC. This trial will provide valuable information on whether similar improvements can be achieved with an alternative type of taxane. Both the IMpassion 130 and IMpassion 131 trials excluded patients with early relapse (disease progression after prior chemotherapy within 12 months for early breast cancer). However, IMpassion 132 (NCT03371017) focuses on patients with early relapsing $\mathrm{TNBC}$ and evaluates the efficacy and safety of combining atezolizumab with non-taxane (gemcitabine plus carboplatin or capecitabine alone). ${ }^{89}$ This trial should indicate whether similar improvements can be achieved with an alternative chemotherapy regimen in patients with early relapsing TNBC.

In addition, ICI plus targeted drugs also demonstrated a feasible clinical efficacy for advanced TNBC. The KEYNOTE 162 trial evaluated the efficacy and safety of pembrolizumab plus niraparib (a PARP inhibitor) in advanced TNBC. The ORR of the overall population was 29\%. Particularly, the BRCA mutation subgroup obtained a quite high ORR of $67 \%$. Patients with PD-L1+ $(>1 \%)$ had a higher ORR than PD-L1- ones $(33 \%$ and $15 \%$, respectively). Moreover, $50 \%$ of patients had grade $\geq 3$ TRAEs, thrombocytopenia, and anemia. ${ }^{90}$ Liu et al initially reported the antitumor activity of anti-PD-1 antibody plus antiangiogenic for advanced TNBC. Patients received camrelizumab and apatinib oral $250 \mathrm{mg}$ continuous dosing (day [d] 1-d14) or intermittent dosing (d1-d7). In the apatinib continuous dosing group, the ORR was $43.3 \%$, while no objective response was found in the apatinib intermittent dosing group. TRAEs in the two groups were manageable. Therefore, combination treatment of camrelizumab and apatinib continuous dosing demonstrated favorable antitumor activity and good tolerance for advanced TNBC. Additionally, this trial found that, in 
Table 4 Clinical Trials: Immunotherapy of Advanced TNBC

\begin{tabular}{|c|c|c|c|c|c|c|c|c|}
\hline \multirow[t]{2}{*}{ Trial } & \multirow[t]{2}{*}{ Phase } & \multirow[t]{2}{*}{ Treatment } & \multirow[t]{2}{*}{$\begin{array}{l}\text { Type of } \\
\text { Drug }\end{array}$} & \multirow[t]{2}{*}{ ORR } & \multirow[t]{2}{*}{$\begin{array}{l}\text { mPFS } \\
\text { (Months) }\end{array}$} & \multirow[t]{2}{*}{$\begin{array}{l}\text { mOS } \\
\text { (Months) }\end{array}$} & \multicolumn{2}{|c|}{$\begin{array}{l}\text { Incidence of } \\
\text { TRAEs }\end{array}$} \\
\hline & & & & & & & Total & Grade $\geq 3$ \\
\hline KEYNOTE-0I 2 & $\mathrm{Ib}$ & Pembro & Anti-PD-I & $18.5 \%$ & NR & NR & $56.3 \%$ & $15.6 \%$ \\
\hline KEYNOTE-028 & $\mathrm{Ib}$ & Pembro & Anti-PD-I & $12.0 \%$ & 1.8 & 8.6 & $64.0 \%$ & $16.0 \%$ \\
\hline $\begin{array}{l}\text { KEYNOTE-086 } \\
\text { cohort A }\end{array}$ & 2 & Pembro & Anti-PD-I & $5.3 \%$ & 2.0 & 9.0 & $60.6 \%$ & $12.9 \%$ \\
\hline $\begin{array}{l}\text { KEYNOTE-086 } \\
\text { cohort B }\end{array}$ & 2 & Pembro & Anti-PD-I & $21.4 \%$ & 2.1 & 18.0 & $63.1 \%$ & $<1 \%$ \\
\hline $\begin{array}{l}\text { JAVELIN solid } \\
\text { tumor }\end{array}$ & $\mathrm{Ib}$ & Avelumab & Anti-PD-I & $5.2 \%$ & NR & NR & $68.5 \%$ & $13.7 \%$ \\
\hline NCTOI 375842 & I & Atezo & Anti-PD-LI & $\begin{array}{l}24.0 \% \\
\text { (first-line) }\end{array}$ & $\mathrm{I} .4$ & 17.6 & $63.0 \%$ & $11.0 \%$ \\
\hline IMpassion I 30 & 3 & $\begin{array}{l}\text { Atezo + nab-P vs } \\
\text { Placebo + nab-P }\end{array}$ & $\begin{array}{l}\text { Anti-PD-LI } \\
+ \text { Chemo }\end{array}$ & NR & 7.2 vs 5.5 & $\begin{array}{l}21.3 \mathrm{vs} \\
17.6\end{array}$ & $\begin{array}{l}99.3 \% \text { vs } \\
97.9 \%\end{array}$ & $\begin{array}{l}48.7 \% \\
\text { vs. } 42.2 \%\end{array}$ \\
\hline $\begin{array}{l}\text { IMpassion I } 30 \\
\text { updated }\end{array}$ & & & & & & $\begin{array}{l}21.0 \mathrm{vs} \\
18.7\end{array}$ & $\begin{array}{l}97.0 \% v s \\
94.0 \%\end{array}$ & $\begin{array}{l}54.0 \% \text { vs } \\
38.0 \%\end{array}$ \\
\hline NCTOI 633970 & $\mathrm{Ib}$ & Atezo + nab-P & $\begin{array}{l}\text { Anti-PD-LI } \\
+ \text { Chemo }\end{array}$ & $39.4 \%$ & 5.5 & 14.7 & $100 \%$ & $73.0 \%$ \\
\hline KEYNOTE-I 62 & 2 & Pembro + Niraparib & $\begin{array}{l}\text { Anti-PD-I + } \\
\text { PARPi }\end{array}$ & $\begin{array}{l}67.0 \% \\
\text { (BRCAmut) }\end{array}$ & 8.1 & NR & $98.2 \%$ & $50.0 \%$ \\
\hline NCT03394287 & 2 & $\begin{array}{l}\text { Camre + Apatinib dI-14 vs } \\
\text { Camre + Apatinib dl-7 }\end{array}$ & $\begin{array}{l}\text { Anti-PD-I + } \\
\text { Antiangi }\end{array}$ & $43.3 \%$ vs 0 & 3.7 vs 1.9 & NR & $\begin{array}{l}100 \% \text { VS } \\
90 \%\end{array}$ & $\begin{array}{l}26.7 \% \text { vs } \\
20.0 \%\end{array}$ \\
\hline
\end{tabular}

Abbreviations: mPFS, median progression-free survival; mOS, overall survival; Pembro, pembrolizumab; Atezo, atezolizumab; nab-P, nab-paclitaxel; Chemo, chemotherapy; Camre, camrelizumab; PARPi, PARP inhibitor; Antiangi, antiangiogenesis.

the continuous dosing group, TILs $>10 \%$ were associated with higher ORR and favorable PFS (Table 4). ${ }^{91}$

\section{PD-LI as a Biomarker}

The JAVELIN Solid Tumor trial was designed to evaluate the efficacy and safety of avelumab (an anti-PD-L1 antibody) in patients with advanced breast cancer. The trial found that antitumor efficacy of avelumab was not related to the amount of PD-L1 expression in tumor cells but was associated with the amount of PD-L1 expression in ICs. In patients with PD-L1+ or PD-L1- ICs (10\% threshold), the ORR was $16.7 \%$ versus $1.6 \%$ in the overall group ( $\mathrm{p}=0.039$ ) and $22.2 \%$ versus $2.6 \%$ in the TNBC subgroup.${ }^{83}$ However, another study on atezolizumab for advanced TNBC found a different relationship between the threshold of PD-L1 ICs and antitumor efficacy. Patients with PD-L1 expression of at least $1 \%$ ICs had higher ORRs than those with $<1 \%$ ICs ( $12 \%$ and 0 , respectively). High levels of
PD-L1+ ICs (>10\%) were independently associated with higher ORR. ${ }^{81}$ As mentioned above, the IMpassion 130 trial suggested that patients who received atezolizumab plus nab-paclitaxel with PD-L1 ICs $>1 \%$ had a longer PFS than the overall group. ${ }^{86}$ The KEYNOTE 162 trial suggested that patients who received pembrolizumab plus niraparib had higher ORR with PD-L1 ICs $>1 \%$ than those with PD-L1 ICs $<1 \% .^{90}$ Therefore, PD-L1 expression in ICs might be related to the antitumor efficacy of PD-1/PDL1 inhibitors. Compared to PD-L1- patients, PD-L1+ patients are more sensitive to PD-1/PD-L1 inhibitor treatment, and the threshold was generally deemed as $1 \%$. However, the threshold of PD-L1 among different test kits may vary. It is noteworthy that Chinese scholars have revealed that nucleophosmin (NPM1) binds to the PD-L1 promoter through its nucleic acid binding domain and activated PD-L1 transcription in TNBC cells. They also demonstrated that PARP1 interacts with the binding 
domain of NPM1, thus suppressing PD-L1 transcription, implying that using PARP1 inhibitors for patients with PD-L1- TNBC will exert a better antitumor effect with anti-PD-L1 therapy. ${ }^{92}$ However, further studies are needed to verify the efficacy of PARP1 inhibitors in PD-L1TNBC patients.

\section{Chemotherapy}

The fourth ESO-ESMO-ABC guideline recommends sequential single-agent chemotherapy as the primary choice for advanced breast cancer. Combination treatment is mainly used for rapid progression, severe visceral metastases, or rapid symptom control. Anthracycline- and taxane-based chemotherapy is usually recommended as the first-line treatment for HER2-negative advanced breast cancer. If patients received prior anthracycline and taxane treatment and do not require combination therapy, monotherapy, such as capecitabine, vinorelbine, and eribulin, are the preferred options. ${ }^{93}$ However, inevitably, some patients are resistant to anthracycline and taxane or do not respond to conventional chemotherapy drugs. Therefore, it is necessary to discuss new strategies to optimize current chemotherapy regimens.

\section{Eribulin}

Eribulin is a tubulin polymerization inhibitor. Study 305 first demonstrated that eribulin prolonged OS compared with TPC in advanced breast cancer. Patients received 2-5 previous chemotherapy regimens, including anthracycline and taxane. The median OS of two groups was 13.1 months and 10.6 months, respectively (HR, 0.81; 95\% CI, $0.66-0.99 ; p=0 \cdot 041)$. ORR was also significantly improved in the eribulin group versus the TPC group ( $12 \%$ and $5 \%$, respectively; $p=0.002$ ). The most common TRAEs in the two groups were fatigue and neutropenia. Incidences of grade $\geq 3$ TRAEs in the two groups were similar. Grade $\geq 3$ TRAEs in the eribulin group were mainly neutropenia and peripheral neuropathy. These TRAEs could be managed by delaying, reducing dosing, and symptomatic treatment. ${ }^{94}$ Study 301 was designed to compare eribulin with capecitabine in advanced breast cancer. ${ }^{95}$ Although no evidence has shown that eribulin was superior to capecitabine in PFS and OS, integration studies 305 and 301 found that eribulin significantly prolonged OS than TPC or capecitabine in advanced TNBC (12.4 months and 8.1 months, respectively; $\mathrm{p}<0.01) .{ }^{96}$ In addition, compared to vinorelbine, eribulin significantly improved PFS $(\mathrm{p}=0.036)$ and ORR $(\mathrm{p}<0.001)$ in advanced breast cancer. The incidence of TRAEs caused by eribulin was lower than that caused by vinorelbine. Subgroup analysis showed that improvement in PFS was also observed with eribulin over vinorelbine in advanced TNBC. ${ }^{97}$ Overall, eribulin demonstrated favorable efficacy and safety in advanced TNBC, providing a new chemotherapy option for advanced refractory TNBC.

\section{Utidelone}

Utidelone is a genetically engineered epothilone analogue, also known as a novel non-taxane tubulin polymerization inhibitor. A study was designed to compare the efficacy and safety of utidelone plus capecitabine with the efficacy and safety of capecitabine alone in patients with breast cancer who had disease progression after prior anthracycline and taxane chemotherapy. The median PFS was 8.44 months in the combination therapy group and 4.27 months in the monotherapy group (HR, 0.46; 95\% CI, 0.36-0.59; p<0.0001). The ORR was $40.4 \%$ in the combination therapy group and $21.5 \%$ in the monotherapy group $(p=0 \cdot 0002)$. Similarly, improvement in PFS was also observed in the combination therapy group over the monotherapy group in the TNBC subgroup. The final result of OS has not been reported. The most common serious TRAE in the combination therapy group was peripheral neuropathy, which was manageable through delaying, reducing dosing, and symptomatic treatment. $^{98}$ This study demonstrated the superiority of utidelone plus capecitabine versus capecitabine alone for either advanced breast cancer or advanced TNBC.

\section{Conclusions}

TNBC is a highly aggressive and heterogeneous subtype of breast $^{\text {cancer. }}{ }^{99,100}$ In the targeted therapy of advanced TNBC, patients carrying germline BRCA1/2 mutation can profit from PARP inhibitors, such as olaparib and talazoparib. AKT inhibitors, ipatasertib, and capivasertib demonstrate efficacy and safety in advanced TNBC. In the PIK3CA/AKT1/PTEN-altered subgroup, the antitumor activity was more significant. AR inhibitors (bicalutamide, enzalutamide, and abiraterone acetate) exhibit antitumor activity and good tolerance in patients with AR+. Optimized bevacizumab-containing regimens resulted in satisfactory outcomes. In the immunotherapy of advanced TNBC, progress is mainly reflected in PD-1/PD-L1 inhibitors. Atezolizumab plus nab-paclitaxel, pembrolizumab plus niraparib, and camrelizumab plus apatinib continuous dosing demonstrated favorable efficacy and safety in advanced TNBC. Patients with BRCA mutation showed more 
significant efficacy from ICI plus PARP inhibitors, such as pembrolizumab plus niraparib. Overall, ICI combination therapy exhibits more significant clinical efficacy than ICI monotherapy, corresponding to a higher incidence of TRAEs in advanced TNBC. PD-L1 as a biomarker in immunotherapy has a certain value in predicting the efficacy of PD-1/PDL1 inhibitors. Taken together, the abovementioned information suggests that it is important to test valid targets and biomarkers for precise and individualized treatment of TNBC. In the chemotherapy of advanced TNBC, eribulin is superior to $\mathrm{TPC} /$ capecitabine/vinorelbine. Combination treatment of utidelone and capecitabine is superior to capecitabine alone. Eribulin and utidelone provide new chemotherapy options for patients with refractory TNBC.

\section{Ethical Statements}

No ethical approval required due to the non-interventional nature of the study. All tables are original.

\section{Acknowledgments}

This research did not receive any grant from funding agencies.

\section{Disclosure}

The authors report no conflicts of interest in this work.

\section{References}

1. Siegel RL, Miller KD, Jemal A. Cancer statistics, 2020. CA Cancer J Clin. 2020;70(1):7-30.

2. Lehmann BD, Bauer JA, Chen X, et al. Identification of human triplenegative breast cancer subtypes and preclinical models for selection of targeted therapies. J Clin Invest. 2011;121(7):2750-2767.

3. Bauer KR, Brown M, Cress RD, Parise CA, Caggiano V. Descriptive analysis of estrogen receptor (ER)-negative, progesterone receptor (PR)-negative, and HER2-negative invasive breast cancer, the socalled triple-negative phenotype: a population-based study from the California cancer Registry. Cancer. 2007;109(9):1721-1728. doi: $10.1002 /$ cncr. 22618

4. Carey LA, Dees EC, Sawyer L, et al. The triple negative paradox: primary tumor chemosensitivity of breast cancer subtypes. Clin Cancer Res. 2007;13(8):2329-2334.

5. Vaz-Luis I, Ottesen RA, Hughes ME, et al. Outcomes by tumor subtype and treatment pattern in women with small, node-negative breast cancer: a multi-institutional study. $J$ clin oncol. 2014;32 (20):2142-2150.

6. Delaloge S, Ezzalfani M, Dieras V, et al. Evolution of overall survival according to year of diagnosis (2008-2014) and subtypes, among 16703 metastatic breast cancer (MBC) patients included in the reallife "ESME" cohort. J Clin Oncol. 2017;35:6.

7. Basho RK, Gilcrease M, Murthy RK, et al. Targeting the PI3K/AKT/ mTOR Pathway for the Treatment of Mesenchymal Triple-Negative Breast Cancer: evidence From a Phase 1 Trial of mTOR Inhibition in Combination With Liposomal Doxorubicin and Bevacizumab. JAMA oncol. 2017;3(4):509-515.
8. Evers B, Helleday T, Jonkers J. Targeting homologous recombination repair defects in cancer. Trends Pharmacol Sci. 2010;31 (8):372-380.

9. Malone KE, Daling JR, Doody DR, et al. Prevalence and predictors of BRCA1 and BRCA2 mutations in a population-based study of breast cancer in white and black American women ages 35 to 64 years. Cancer Res. 2006;66(16):8297-8308.

10. Couch FJ, Hart SN, Sharma P, et al. Inherited mutations in 17 breast cancer susceptibility genes among a large triple-negative breast cancer cohort unselected for family history of breast cancer. $J$ clin oncol. 2015;33(4):304-311. doi:10.1200/JCO.2014. 57.1414

11. Atchley DP, Albarracin CT, Lopez A, et al. Clinical and Pathologic Characteristics of Patients With BRCA -Positive and BRCA -Negative Breast Cancer. J clin oncol. 2008;26(26):42824288. doi:10.1200/JCO.2008.16.6231

12. Anwar M, Aslam HM, Anwar S. PARP inhibitors. Hered Cancer Clin Pract. 2015;13(1):4. doi:10.1186/s13053-014-0024-8

13. Bryant HE, Schultz N, Thomas HD, et al. Specific killing of BRCA2-deficient tumours with inhibitors of poly(ADP-ribose) polymerase. Nature. 2005;434(7035):913-917. doi:10.1038/ nature 03443

14. Ashworth A, Lord CJ. Synthetic lethal therapies for cancer: what's next after PARP inhibitors? Nat Rev Clin Oncol. 2018;15 (9):564-576. doi:10.1038/s41571-018-0055-6

15. Lord CJ, Tutt ANJ, Ashworth A. Synthetic lethality and cancer therapy: lessons learned from the development of PARP inhibitors. Annu Rev Med. 2015;66(1):455-470. doi:10.1146/annurevmed-050913-022545

16. Robson M, Im S-A, Senkus E, et al. Olaparib for Metastatic Breast Cancer in Patients with a Germline BRCA Mutation. $N$ Engl J Med. 2017;377(6):523-533. doi:10.1056/NEJMoa1706450

17. Robson ME, Tung N, Conte P, et al. OlympiAD final overall survival and tolerability results: olaparib versus chemotherapy treatment of physician's choice in patients with a germline BRCA mutation and HER2-negative metastatic breast cancer. Ann Oncol. 2019;30(4):558-566. doi:10.1093/annonc/mdz012

18. Litton JK, Rugo HS, Ettl J, et al. Talazoparib in Patients with Advanced Breast Cancer and a Germline BRCA Mutation. N Engl J Med. 2018;379(8):753-763. doi:10.1056/ NEJMoa1802905

19. Ettl J, Quek RGW, Lee K-H, et al. Quality of life with talazoparib versus physician's choice of chemotherapy in patients with advanced breast cancer and germline BRCA1/2 mutation: patient-reported outcomes from the EMBRACA Phase III trial. Ann Oncol. 2018;29(9):1939-1947. doi:10.1093/annonc/mdy257

20. Turner NC, Telli ML, Rugo HS, et al. A Phase II Study of Talazoparib after Platinum or Cytotoxic Nonplatinum Regimens in Patients with Advanced Breast Cancer and Germline BRCA1/2 Mutations (ABRAZO). Clin Cancer Res. 2019;25(9):2717-2724. doi:10.1158/1078-0432.CCR-18-1891

21. Rodler ET, Kurland BF, Griffin M, et al. Phase I Study of Veliparib (ABT-888) Combined with Cisplatin and Vinorelbine in Advanced Triple-Negative Breast Cancer and/or BRCA Mutation-Associated Breast Cancer. Clin Cancer Res. 2016;22 (12):2855-2864. doi:10.1158/1078-0432.CCR-15-2137

22. Han HS, Diéras V, Robson M, et al. Veliparib with temozolomide or carboplatin/paclitaxel versus placebo with carboplatin/paclitaxel in patients with BRCA1/2 locally recurrent/metastatic breast cancer: randomized phase II study. Ann Oncol. 2018;29(1):154161. doi: $10.1093 / \mathrm{annonc} / \mathrm{mdx} 505$

23. Nuthalapati S, Stodtmann S, Shepherd SP, et al. Exposureresponse analysis to inform the optimal dose of veliparib in combination with carboplatin and paclitaxel in BRCA-mutated advanced breast cancer patients. Cancer Chemother Pharmacol. 2019;84(5):977-986. doi:10.1007/s00280-019-03930-2 
24. Wesolowski R, Stover DG, Lustberg MB, et al. Phase I Study of Veliparib on an Intermittent and Continuous Schedule in Combination with Carboplatin in Metastatic Breast Cancer: A Safety and [18F]-Fluorothymidine Positron Emission Tomography Biomarker Study. oncologist. 2020;25(8). doi:10.1634/theoncologist.2020-0039

25. Ortega MA, Fraile-Martínez O, Asúnsolo Á, Buján J, GarcíaHonduvilla N, Coca S. Signal Transduction Pathways in Breast Cancer: the Important Role of PI3K/Akt/mTOR. J Oncol. 2020;2020:9258396. doi:10.1155/2020/9258396

26. Altomare DA, Testa JR. Perturbations of the AKT signaling pathway in human cancer. Oncogene. 2005;24(50):7455-7464 doi:10.1038/sj.onc. 1209085

27. Nitulescu GM, Margina D, Juzenas P, et al. Akt inhibitors in cancer treatment: the long journey from drug discovery to clinical use (Review). Int J Oncol. 2016;48(3):869-885. doi:10.3892 ijo.2015.3306

28. Network CGA. Comprehensive molecular portraits of human breast tumours. Nature. 2012;490(7418):61-70.

29. Costa RLB, Han HS, Gradishar WJ. Targeting the PI3K/AKT/ mTOR pathway in triple-negative breast cancer: a review. Breast Cancer Res Treat. 2018;169(3):397-406. doi:10.1007/s10549018-4697-y

30. Kim S-B, Dent R, Im S-A, et al. Ipatasertib plus paclitaxel versus placebo plus paclitaxel as first-line therapy for metastatic triplenegative breast cancer (LOTUS): a multicentre, randomised, double-blind, placebo-controlled, phase 2 trial. Lancet Oncol. 2017;18(10):1360-1372. doi:10.1016/S1470-2045(17)30450-3

31. Dent R, Im S-A, Espie M, Blau S, Tan AR, Isakoff SJ. Overall survival (OS) update of the double-blind placebo (PBO)-controlled randomized phase 2 LOTUS trial of first-line ipatasertib (IPAT) + paclitaxel (PAC) for locally advanced/metastatic triplenegative breast cancer (mTNBC).. J Clin Oncol. 2018;36 (15_suppl):2. doi:10.1200/JCO.2018.36.15_suppl.1008

32. Schmid P, Abraham J, Chan S, et al. Capivasertib Plus Paclitaxel Versus Placebo Plus Paclitaxel As First-Line Therapy for Metastatic Triple-Negative Breast Cancer: the PAKT Trial. $J$ clin oncol. 2020;38(5):423-433. doi:10.1200/JCO.19.00368

33. Ni M, Chen Y, Lim E, et al. Targeting androgen receptor in estrogen receptor-negative breast cancer. Cancer Cell. 2011;20 (1):119-131. doi:10.1016/j.ccr.2011.05.026

34. Rahim B, O’Regan R. AR Signaling in Breast Cancer. Cancers. 2017;9(12):3. doi:10.3390/cancers9030021

35. Lehmann BD, Bauer JA, Schafer JM, et al. PIK3CA mutations in androgen receptor-positive triple negative breast cancer confer sensitivity to the combination of PI3K and androgen receptor inhibitors. Breast Cancer Res. 2014;16(4):406. doi:10.1186/ s13058-014-0406-x

36. Davis M, Tripathi S, Hughley R, et al. AR negative triple negative or "quadruple negative" breast cancers in African American women have an enriched basal and immune signature. PLoS One. 2018;13(6):e0196909. doi:10.1371/journal.pone.0196909

37. Vera-Badillo FE, Templeton AJ, de Gouveia P, et al. Androgen receptor expression and outcomes in early breast cancer: a systematic review and meta-analysis. J Natl Cancer Inst. 2014;106 (1):djt319.

38. Barton VN, D'Amato NC, Gordon MA, Christenson JL, Elias A, Richer JK. Androgen Receptor Biology in Triple Negative Breast Cancer: a Case for Classification as AR+ or Quadruple Negative Disease. Horm Cancer. 2015;6(5-6):206-213.

39. Hon JD, Singh B, Sahin A, et al. Breast cancer molecular subtypes: from TNBC to QNBC. Am J Cancer Res. 2016;6(9):1864-1872.

40. Anestis A, Karamouzis MV, Dalagiorgou G, Papavassiliou AG. Is androgen receptor targeting an emerging treatment strategy for triple negative breast cancer? Cancer Treat Rev. 2015;41(6):547553.
41. Yang F, Shen Y, Zhang W, et al. An androgen receptor negatively induced long non-coding RNA ARNILA binding to miR-204 promotes the invasion and metastasis of triple-negative breast cancer. Cell Death Differ. 2018;25(12):2209-2220.

42. Gucalp A, Tolaney S, Isakoff SJ, et al. Phase II trial of bicalutamide in patients with androgen receptor-positive, estrogen receptor-negative metastatic Breast Cancer. Clin Cancer Res. 2013;19 (19):5505-5512.

43. Taplin ME, Montgomery B, Logothetis CJ, et al. Intense androgen-deprivation therapy with abiraterone acetate plus leuprolide acetate in patients with localized high-risk prostate cancer: results of a randomized phase II neoadjuvant study. J clin oncol. 2014;32 (33):3705-3715

44. Attard G, Reid AH, Yap TA, et al. Phase I clinical trial of a selective inhibitor of CYP17, abiraterone acetate, confirms that castration-resistant prostate cancer commonly remains hormone driven. J clin oncol. 2008;26(28):4563-4571.

45. Bonnefoi H, Grellety $\mathrm{T}$, Tredan $\mathrm{O}$, et al. A phase II trial of abiraterone acetate plus prednisone in patients with triple-negative androgen receptor positive locally advanced or metastatic breast cancer (UCBG 12-1). Ann Oncol. 2016;27(5):812-818.

46. Traina TA, Miller K, Yardley DA, et al. Enzalutamide for the Treatment of Androgen Receptor-Expressing Triple-Negative Breast Cancer. J clin oncol. 2018;36(9):884-890.

47. Curigliano G, Pivot X, Cortés J, et al. Randomized phase II study of sunitinib versus standard of care for patients with previously treated advanced triple-negative breast cancer. Breast. 2013;22 (5):650-656.

48. Miller K, Wang M, Gralow J, et al. Paclitaxel plus bevacizumab versus paclitaxel alone for metastatic breast cancer. $N$ Engl J Med. 2007;357(26):2666-2676.

49. Miles DW, Diéras V, Cortés J, Duenne AA, Yi J, O’Shaughnessy J. First-line bevacizumab in combination with chemotherapy for HER2negative metastatic breast cancer: pooled and subgroup analyses of data from 2447 patients. Ann Oncol. 2013;24(11):2773-2780.

50. Saloustros E, Nikolaou M, Kalbakis K, et al. Weekly Paclitaxel and Carboplatin Plus Bevacizumab as First-Line Treatment of Metastatic Triple-Negative Breast Cancer. A Multicenter Phase II Trial by the Hellenic Oncology Research Group. Clin Breast Cancer. 2018;18(1):88-94.

51. Symonds L, Linden H, Gadi V, et al. Combined Targeted Therapies for First-line Treatment of Metastatic Triple Negative Breast CancerA Phase II Trial of Weekly Nab-Paclitaxel and Bevacizumab Followed by Maintenance Targeted Therapy With Bevacizumab and Erlotinib. Clin Breast Cancer. 2019;19(2):e283-e296.

52. Wang Y, Wu S, Zhu X, et al. LncRNA-encoded polypeptide ASRPS inhibits triple-negative breast cancer angiogenesis. $J$ Exp Med. 2020;217:3.

53. Pan T, Zhou D, Shi Z, et al. Centromere protein U (CENPU) enhances angiogenesis in triple-negative breast cancer by inhibiting ubiquitin-proteasomal degradation of COX-2. Cancer Lett. 2020;482:102-111

54. Li H, Xu F, Gao G, et al. Hydrogen sulfide and its donors: novel antitumor and antimetastatic therapies for triple-negative breast cancer. Redox Biol. 2020;34:101564.

55. Chaudhary P, Gibbs LD, Maji S, Lewis CM, Suzuki S, Vishwanatha JK. Correction to: serum exosomal-annexin A2 is associated with African-American triple-negative breast cancer and promotes angiogenesis. Breast Cancer Res. 2020;22(1):31.

56. Dean JL, Thangavel C, McClendon AK, Reed CA, Knudsen ES. Therapeutic CDK4/6 inhibition in breast cancer: key mechanisms of response and failure. Oncogene. 2010;29(28):4018-4032.

57. Yamamoto T, Kanaya N, Somlo G, Chen S. Synergistic anticancer activity of CDK4/6 inhibitor palbociclib and dual mTOR kinase inhibitor MLN0128 in pRb-expressing ER-negative breast cancer. Breast Cancer Res Treat. 2019;174(3):615-625. 
58. Pearson A, Proszek P, Pascual J, et al. Inactivating NF1 Mutations Are Enriched in Advanced Breast Cancer and Contribute to Endocrine Therapy Resistance. Clin Cancer Res. 2020;26 (3):608-622.

59. Ji W, Shi Y, Wang X, et al. Combined Androgen receptor blockade overcomes the resistance of breast cancer cells to palbociclib. Int J Biol Sci. 2019;15(3):522-532.

60. Gucalp A, Edelweiss M, Patil S, et al. Phase I/II trial of palbociclib in combination with bicalutamide for the treatment of androgen receptor (AR) plus metastatic breast cancer (MBC). Cancer Res. 2018;78(4):1.

61. Johnstone CN, Pattison AD, Harrison PF, et al. FGF13 promotes metastasis of triple-negative breast cancer. Int $j$ Cancer. 2020;147 (1):230-243.

62. Chew NJ, Nguyen EV, Su SP, et al. FGFR3 signaling and function in triple negative breast cancer. Cell Commun Signaling. 2020;18 (1): 13 .

63. Pines M, Levi O, Genin O, et al. Elevated Expression of Moesin in Muscular Dystrophies. Am J Pathol. 2017;187(3):654-664.

64. Qin Y, Chen W, Jiang G, et al. Interfering MSN-NONO complexactivated CREB signaling serves as a therapeutic strategy for triple-negative breast cancer. Sci Adv. 2020;6(8):eaaw9960.

65. Turner N, Moretti E, Siclari O, et al. Targeting triple negative breast cancer: is p53 the answer? Cancer Treat Rev. 2013;39 (5):541-550.

66. Aarts M, Sharpe R, Garcia-Murillas I, et al. Forced mitotic entry of S-phase cells as a therapeutic strategy induced by inhibition of WEE1. Cancer Discov. 2012;2(6):524-539.

67. Karnitz LM, Molecular Pathways: ZL. Targeting ATR in Cancer Therapy. Clin Cancer Res. 2015;21(21):4780-4785.

68. Jin J, Fang H, Yang F, et al. Combined Inhibition of ATR and WEE1 as a Novel Therapeutic Strategy in Triple-Negative Breast Cancer. Neoplasia. 2018;20(5):478-488.

69. Miyazaki T, Tanaka S, Sanjay A, Baron R. The role of c-Src kinase in the regulation of osteoclast function. Modern Rheumatology. 2006;16(2):68-74.

70. Heilmann T, Rumpf AL, Roscher M, et al. Dasatinib prevents skeletal metastasis of osteotropic MDA-MB-231 cells in a xenograft mouse model. Arch Gynecol Obstet. 2020;301(6):14931502.

71. Canonici A, Browne AL, Ibrahim MFK, et al. Combined targeting EGFR and SRC as a potential novel therapeutic approach for the treatment of triple negative breast cancer. Ther Adv Med Oncol. 2020;12:1758835919897546.

72. Corrales-Sánchez V, Mdm N-L, Nieto-Jiménez C, et al. Pharmacological screening and transcriptomic functional analyses identify a synergistic interaction between dasatinib and olaparib in triple-negative breast cancer. J Cell Mol Med. 2020;24 (5):3117-3127.

73. Bellat V, Verchère A, Ashe SA, Law B. Transcriptomic insight into salinomycin mechanisms in breast cancer cell lines: synergistic effects with dasatinib and induction of estrogen receptor $\beta$. BMC Cancer. 2020;20(1):661.

74. Topalian SL, Drake CG, Pardoll DM. Immune checkpoint blockade: a common denominator approach to cancer therapy. Cancer Cell. 2015;27(4):450-461.

75. Luen S, Virassamy B, Savas P, Salgado R, Loi S. The genomic landscape of breast cancer and its interaction with host immunity. Breast. 2016;29:241-250.

76. Dill EA, Gru AA, Atkins KA, et al. PD-L1 Expression and Intratumoral Heterogeneity Across Breast Cancer Subtypes and Stages: an Assessment of 245 Primary and 40 Metastatic Tumors. Am J Surg Pathol. 2017;41(3):334-342.

77. Mittendorf EA, Philips AV, Meric-Bernstam F, et al. PD-L1 expression in triple-negative breast cancer. Cancer Immunol Res. 2014;2(4):361-370.
78. Sabatier R, Finetti P, Mamessier E, et al. Prognostic and predictive value of PDL1 expression in breast cancer. Oncotarget. 2015;6(7):5449-5464.

79. Nanda R, Chow LQ, Dees EC, et al. Pembrolizumab in Patients With Advanced Triple-Negative Breast Cancer: phase Ib KEYNOTE-012 Study. J clin oncol. 2016;34(21):2460-2467.

80. Adams S, Loi S, Toppmeyer D, et al. Pembrolizumab monotherapy for previously untreated, PD-L1-positive, metastatic triplenegative breast cancer: cohort B of the phase II KEYNOTE-086 study. Ann Oncol. 2019;30(3):405-411.

81. Emens LA, Cruz C, Eder JP, et al. Long-term Clinical Outcomes and Biomarker Analyses of Atezolizumab Therapy for Patients With Metastatic Triple-Negative Breast Cancer: A Phase 1 Study. JAMA oncol. 2019;5(1):74-82.

82. Adams S, Schmid P, Rugo HS, et al. Pembrolizumab monotherapy for previously treated metastatic triple-negative breast cancer: cohort A of the phase II KEYNOTE-086 study. Ann Oncol. 2019;30(3):397-404.

83. Dirix LY, Takacs I, Jerusalem G, et al. Avelumab, an anti-PD-L1 antibody, in patients with locally advanced or metastatic breast cancer: a phase 1b JAVELIN Solid Tumor study. Breast Cancer Res Treat. 2018;167(3):671-686.

84. Rugo HS, Delord JP, Im SA, et al. Safety and Antitumor Activity of Pembrolizumab in Patients with Estrogen Receptor-Positive/ Human Epidermal Growth Factor Receptor 2-Negative Advanced Breast Cancer. Clin Cancer Res. 2018;24(12):2804-2811.

85. Adams S, Gatti-Mays ME, Kalinsky K, et al. Current Landscape of Immunotherapy in Breast Cancer: A Review. JAMA oncol. 2019;1:123.

86. Schmid P, Adams S, Rugo HS, et al. Atezolizumab and NabPaclitaxel in Advanced Triple-Negative Breast Cancer. $N$ Engl J Med. 2018;379(22):2108-2121.

87. Schmid P, Rugo HS, Adams S, et al. Atezolizumab plus nabpaclitaxel as first-line treatment for unresectable, locally advanced or metastatic triple-negative breast cancer (IMpassion130): updated efficacy results from a randomised, double-blind, placebo-controlled, phase 3 trial. Lancet Oncol. 2020;21(1):44-59.

88. Adams S, Diamond JR, Hamilton E, et al. Atezolizumab Plus nab-Paclitaxel in the Treatment of Metastatic Triple-Negative Breast Cancer With 2-Year Survival Follow-up: A Phase 1b Clinical Trial. JAMA oncol. 2019;5(3):334-342.

89. Cortés J, André F, Gonçalves A, et al. IMpassion132 Phase III trial: atezolizumab and chemotherapy in early relapsing metastatic triple-negative breast cancer. Future Oncol. 2019;15(17):1951-1961.

90. Vinayak S, Tolaney SM, Schwartzberg LS, et al. TOPACIO/ Keynote-162: niraparib plus pembrolizumab in patients (pts) with metastatic triple-negative breast cancer (TNBC), a phase 2 trial. J Clin Oncol. 2018;36(15):2.

91. Liu J, Liu Q, Li Y, et al. Efficacy and safety of camrelizumab combined with apatinib in advanced triple-negative breast cancer: an open-label phase II trial. J Immunother Cancer. 2020;8:1.

92. Qin G, Wang X, Ye S, et al. NPM1 upregulates the transcription of PD-L1 and suppresses T cell activity in triple-negative breast cancer. Nat Commun. 2020;11(1):1669.

93. Cardoso F, Senkus E, Costa A, et al. 4th ESO-ESMO International Consensus Guidelines for Advanced Breast Cancer (ABC 4)†. Ann Oncol. 2018;29(8):1634-1657.

94. Cortes J, O’Shaughnessy J, Loesch D, et al. Eribulin monotherapy versus treatment of physician's choice in patients with metastatic breast cancer (EMBRACE): a phase 3 open-label randomised study. Lancet. 2011;377(9769):914-923.

95. Kaufman PA, Awada A, Twelves C, et al. Phase III open-label randomized study of eribulin mesylate versus capecitabine in patients with locally advanced or metastatic breast cancer previously treated with an anthracycline and a taxane. $J$ clin oncol. 2015;33(6):594-601. 
96. Pivot X, Marmé F, Koenigsberg R, Guo M, Berrak E, Wolfer A. Pooled analyses of eribulin in metastatic breast cancer patients with at least one prior chemotherapy. Ann Oncol. 2016;27 (8):1525-1531.

97. Yuan $\mathrm{P}, \mathrm{Hu} \mathrm{X}$, Sun T, et al. Eribulin mesilate versus vinorelbine in women with locally recurrent or metastatic breast cancer: A randomised clinical trial. Eur $j$ Cancer. 2019;112:57-65.

98. Zhang P, Sun T, Zhang Q, et al. Utidelone plus capecitabine versus capecitabine alone for heavily pretreated metastatic breast cancer refractory to anthracyclines and taxanes: a multicentre, open-label, superiority, phase 3, randomised controlled trial. Lancet Oncol. 2017;18(3):371-383.
99. Yang F, Wang Y, Li Q, et al. Intratumor heterogeneity predicts metastasis of triple-negative breast cancer. Carcinogenesis. 2017;38(9):900-909.

100. Yang F, Cao L, Sun Z, et al. Evaluation of Breast Cancer Stem Cells and Intratumor Stemness Heterogeneity in Triple-negative Breast Cancer as Prognostic Factors. Int J Biol Sci. 2016;12 (12):1568-1577. doi:10.7150/ijbs. 16874

\section{Publish your work in this journal}

Cancer Management and Research is an international, peer-reviewed open access journal focusing on cancer research and the optimal use of preventative and integrated treatment interventions to achieve improved outcomes, enhanced survival and quality of life for the cancer patient.
The manuscript management system is completely online and includes a very quick and fair peer-review system, which is all easy to use. Visit http://www.dovepress.com/testimonials.php to read real quotes from published authors. 